\title{
R_transport_matrices of the Fast Extraction Beam (FEB) of the AGS, and Beam Parameters at the Starting point of the AtR Line
}

\author{
N. Tsoupas, W.W. MacKay, T. Satogata, W. Glenn, L. Ahrens, K. Brown, \\ C. Gardner, S. Tanaka
}



Collider-Accelerator Department Brookhaven National Laboratory Upton, NY 11973

Notice: This document has been authorized by employees of Brookhaven Science Associates, LLC under Contract No. DE-AC02-98CH10886 with the U.S. Department of Energy. The United States Government retains a non-exclusive, paidup, irrevocable, world-wide license to publish or reproduce the published form of this document, or allow others to do so, for United States Government purposes. 


\title{
R_transport_matrices of the Fast Extraction Beam (FEB) of the AGS, and Beam Parameters at the Starting point of the AtR Line
}

\author{
N. Tsoupas, W.W. MacKay, T. Satogata, W. Glenn, L. Ahrens, K. Brown, C. Gardner, \\ Sanki Tanaka, others?
}

\begin{abstract}
:
As part of the task to improve and further automate the "AtR BPM Application" we provide the theoretically calculated R_transport_matrices for the following beam line sections, which are shown schematically in Figure 1:

a) the Fast Extraction Beam section (FEB) of the AGS synchrotron. The FEB section starts at the middle of the G10_kicker and ends at the middle of the H10_septum.

b) the Drift Extraction Channel (DEC) section of the AGS synchrotron. The DEC section starts at the middle of the H10_septum, continues along the fringe field region of the $\mathrm{H} 11, \mathrm{H} 12$, and $\mathrm{H} 13$ AGS main magnets, and ends at the starting point of the AtR line.
\end{abstract}

The knowledge of these R_transport_matrices are needed in order to calculate the beam parameters at the beginning of the AtR line, which, in turn, are required to calculate the magnet settings of the U_line, that match the U_line into the W_line.

Also by incorporating these R_matrices into the model of the AtR line, the G10 kicker and the H10 septum are included in the AtR model therefore one can investigate any "jitter" of either the G10_kicker or H10_septum by looking at the trajectory of the beam in the AtR line.

\section{INTRODUCTION}

The AGS to RHIC (AtR) beam transport line, transports the extracted beam from the AGS to RHIC. As we mentioned earlier, and we repeat here, there are two basic requirements that the AtR beam transport line should satisfy; one is the "correct beam optics" of the AtR line, which relies on the proper magnet settings of the AtR line, and the other requirement is the stable beam trajectory in the AtR line. The stable beam trajectory relies on the good stability of the magnetic field generated by the AtR magnets and the various extraction devices, like the G10_kicker and H10_septum.

The first requirement, of a "correct beam optics", ensures proper matching of the transported beam with the lattice of the AtR line, especially the $20^{\circ}$ bend of the W_section, which consists of a FODO lattice, and the $90^{\circ}$ bends of the X and Y sections of the AtR line, which are also consist of FODO lattic es. This proper beam matching will eliminate beam losses along the AtR line and also ascertain the correct beam parameters at the injection point of RHIC. This requirement, of the "correct magnet settings", can be tested by the use of the "AtR BPM Application" code.

We have to inform the reader that the magnet settings of the AtR line are based on:

a) the values of the beam parameters at the beginning of the AtR line.

b) the beam constraints imposed along the AtR line,

c) and the beam matching of the various beam sections of the AtR line, namely; matching of U_line to the W_line 
matching of the $\mathrm{W} \_$line to the $\mathrm{X}$ and $\mathrm{Y}$ lines.

matching of the $\mathrm{X}$ and $\mathrm{Y}$ lines to the Injection points of RHIC.

These beam parameters at the beginning of the AtR line can be calculated theoretically and be compared with the measured ones. Since the theoretically calculated beam parameters are relatively accurate, this comparison will serve as a test that the measured beam parameters and beam emittance of in the AtR line are correct. In case that there is a discrepancy between the theoretical and experimental values of the beam parameters, it will indicate that either, the device which measures the beam parameters is malfunctioning or the beam extraction setup of the AGS has a major flaw.

The second requirement of the AtR line, which is the repeatability of the beam trajectory of the transported beam bunches, will ensure good beam injection repeatability into RHIC. Both of these requirements; the correct magnet settings, and the repeatability of the beam trajectory, can be tested, and subsequently satisfied, by the use of the automated version of the "AtR_BPM application".

This document will be dealing with:

a) the justification to provide the present write up for the theoretically calculated

R_transfer_matrices mentioned earlier, and the beam parameters at the beginning of the AtR line

b) the step by step procedure utilized to calculate the R_transfer_matrices, and the beam parameters at the beginning of the AtR line.

c) Presentation of tables with the R_matric es of the "FEB" and "DEC" lines, and of the beam parameters at the beginning of the AtR line for various beam extraction settings of the AGS.

Why do we need a specific write-up for the calculation of the $R$ _Matrices? Can one simply obtain these R_matrices from a "MAD_model" of the AGS and AtR lines?

A straightforward way to calculate the R_matrices of the various line sections of the FEB_AtR_line, is to generate a MAD model of the FEB_AtR_line, which can be partitioned in three beam lines according to the relation below.

$\{$ FEB_AtR_line $\}<=>$ FEB_section $\}+\{$ DEC_section $\}+\{$ AtR_line $\}$ (See Figure 1$)$

Such a model can be easily generated for the FEB section and the AtR_line, since both lines consist of well described magnetic elements, like dipoles and quadrupoles.

The DEC section, however, is located inside the fringe field of the H11,H12 and H13 AGS main magnets and the R_matrices can only be calculated by the "raytrace" method which requires the knowledge of the magnetic field of the region where the DEC section is located.

Indeed the DEC line section is part of the area, where field maps, over the median plane of the AGS main magnets, have been generated[2]. These field maps can therefore be used to calculate the R_matrices of the DEC section by using the raytrace method. Thus this document provides the R_transport_matrices of the "FEB" and "DEC" sections of the extraction line of the AGS, for various extraction settings of the AGS synchrotron. The R_matrices are calculated by raytracing the extracted beam using the experimentally 
measured field maps [2]. This document provides also the beam parameters at the beginning of the AtR line for various extraction settings of the AGS synchrotron.

\section{Procedure on how to calculate the $R \_$Matrices of the "FEB" and "DEC" Lines}

In this section we provide details on how we calculated of the R_matrices of the FEB and DEC lines (see Fig. 1) of the AGS and also provide details on the calculations of the beam parameters at the beginning of the AtR line.

To remind the reader, the FEB section, shown schematically in Figure 1, starts from the middle of the G10_kicker, which is the first element of the FEB_section and ends at the middle of the H10_septum. The G10_kicker is represented by a "corrector magnet" of zero length, and its effect is included in the calculations of the R_matrix of the FEB_section. The H10_septum is nor included in the FEB_section.

The DEC section, shown schematically in Figure 1, starts from the middle of the H10_septum, which is the first element of the DEC_section, and ends at the starting point of the AtR line. The H10_septum is represented by a "corrector magnet" of zero length, and its effect is included in the calculations of the R_matrix of the DEC_section. Thus the total "FEB_AtR_line" will be represented by the following model:

FEB_AtR_line $=\{$ G10_kicker+FEB_matrix $\}+\{$ H10_septum + DEC_matrix $\}+\{$ AtR_line $\}$

Two methods were used to calculate the R_matrices of the FEB_section; the first method utilizes a "MAD_model" of the AGS ring, and the second method utilizes "Raytracing" through the AGS magnets.

For reasons described above, the calculations of the DEC matrices were performed by the "Raytracing" method only.

\section{Calculation of the "FEB" matrices using a "MAD model" of the AGS ring}

In order to calculate the R_matrices of the FEB section by using the MAD code, we use a MAD model of the AGS that includes the following features:

a) The values of the physical quantities $\mathrm{K} 1$ and $\mathrm{K} 2$, which are required to represent the combined function main magnets of the AGS are incorporated in the MAD model as a function of the beam momentum. This functional dependence of the $\mathrm{K} 1$ and $\mathrm{K} 2$ is referred among the AGS personnel, as the "Ed Bleser's model of the AGS main magnets".

b) The AGS main magnets as they are represented in the "MAD_model" of the AGS, have been artificially split in the middle, and a kicker magnet of zero length has been placed in the middle. These kicker magnets are utilized in the optimization of the G10 and H10 extraction orbit bumps and provide a good approximation for the "back leg windings" settings, of the main magnets which are actually used to generate these local beam orbit bumps. The "back leg windings" are part of the FEB system [3]. In order to provide more accurate description of the local beam bumps of the extraction orbit, (following the optimization of the extraction orbit bumps which utilizes the 
artificial kickers placed in the middle of the main magnets), we replace the strength of each artificial kicker that generated the G10 and H10 local beam bumps, by a magnet error $(\Delta \mathrm{K} 0, \Delta \mathrm{K} 1$, and $\Delta \mathrm{K} 2)$. Subsequently the local orbit bumps are further optimized by "trial and error" of the magnet error $(\Delta \mathrm{K} 0)$. This representation of the artificial kickers magnets by magnet errors is a more accurate description of the back leg windings of the main magnets which are actually used to generate these local orbit bumps of the extracted beam.

c) The strengths of both, the Tune Quadrupoles and Chromaticity Sextupoles are also included in the "MAD" Model of the FEB section.

\section{Procedure to calculate the $R \_$matrices of the FEB section using the "MAD model"}

In this section we describe the step by step procedure to calculate the $R \_$matrices of the FEB section by using the MAD model.

1. Set up AGS for beam extraction using the "MAD_model" of AGS as described above:

1.1 Main magnets of AGS are set to allow beam with the required "momentum at extraction" to circulate . The required "beam momentum at extraction" corresponds to the momentum of the beam which allows the beam to circulate at a radius of $\mathrm{R}_{0}=0.0[\mathrm{~mm}]$. The actual extraction momentum of the beam is achieved when the beam is moved to an outer radius, and also, the local beam extraction bumps "G10" and "H10" are energized.

1.2 The Tune_Quadrupoles and Chromaticity_sextupoles are set to generate the required tunes $\mathrm{Q}_{\mathrm{x}, \mathrm{y}}$ and chromaticities $\xi_{\mathrm{x}, \mathrm{y}}$. These required tunes and chromaticities are the tunes and chromaticities of the AGS synchrotron as measured with the beam just before extraction.

1.3 The beam orbit is placed at the desired radius. At this desired radius the beam momentum is the actual beam momentum that the RHIC synchrotron desires during injection. Also while the beam circulates at the desired radius the local beam bumps G10 and H10 (see item 1.4 below) are energized.

The local beam bumps for fast extraction are set to provide the required $X_{\text {cod }}$ at the middle of the G10_kicker and the middle of the H10_septum [1,3] . The required closed orbit displacements are: $\left(\mathrm{X}_{\mathrm{cod}}\right)_{\delta \mathrm{p} / \mathrm{p}=0}($ at $\mathrm{G} 10)=\sim 61[\mathrm{~mm}]$ and $\left(\mathrm{X}_{\mathrm{cod}}\right)_{\delta \mathrm{p} / \mathrm{p}=0}($ at $\mathrm{H} 10)=\sim 48[\mathrm{~mm}]$. These beam displacements at $\mathrm{G} 10$ and $\mathrm{H} 10$ have been calculated when the fast extraction system of the AGS was designed, and take into account the size of the beam at extraction so that the beam does not interact with any apertures. Also the beam displacements $\left(\mathrm{X}_{\mathrm{cod}}\right)_{\delta \mathrm{p} / \mathrm{p}=0}$ (at G10) and $\left(\mathrm{X}_{\mathrm{cod}}\right) \delta \mathrm{p} / \mathrm{p}=0$ (at H10) should always be $\sim 61[\mathrm{~mm}]$ and $\sim 61[\mathrm{~mm}]$ respectively, independent of the average radius of the circulating beam.

The meaning of the $\delta \mathrm{p} / \mathrm{p}=0.0$ is discussed in the rest of this section. In either the MAD code of the BEAM code, for the beam to be placed at 
the desired extraction radius one must add a momentum $\mathrm{dp} / \mathrm{p}$ to the required "beam momentum at extraction". However in this write up we call the final momentum of the circulating beam at the extraction radius and with the extraction bumps energized as having a $\mathrm{dp} / \mathrm{p}=0.0000$

1.4 The following physical quantities, for the closed beam orbit, are recorded:

i. The beam parameters $\left(\beta_{\mathrm{xy}}, \alpha_{\mathrm{xy}}\right)$ at the location of the G10_kicker.

ii. The $X_{\text {cod }}$ and $X_{\text {cod }}^{\prime}$ at the location of the G10 kicker for two different momenta of the central orbit, $\delta \mathrm{p} / \mathrm{p}=0$ and $\delta \mathrm{p} / \mathrm{p}=0.001$. It is required that $\left(\mathrm{X}_{\mathrm{cod}}\right)_{\delta \mathrm{p} / \mathrm{p}=0}($ at $\mathrm{G} 10)=\sim 61[\mathrm{~mm}]$ and $\left(\mathrm{X}_{\mathrm{cod}}\right)_{\delta \mathrm{p} / \mathrm{p}=0}($ at $\mathrm{H} 10)=\sim 48[\mathrm{~mm}]$.

2. After the orbit is closed, we use the MAD model of the AGS section between the G10_kicker and the H10_septum: this section was defined earlier as the "FEB" section.

2.1 With the magnetic elements of the FEB section set as in item (1.) above and the beam's initial conditions same as those recorded in (1.4 above), we kick the beam with the G10_kicker. The strength of the G10_kicker should be such that the central ray of the beam, referred as "Extracted orbit" in Table 1., should have the required displacement $\left(\mathrm{X}_{\mathrm{cod}}\right)_{\delta \mathrm{p} / \mathrm{p}=0}$, midway of the H10_septum. This central orbit displacement $\left(\mathrm{X}_{\mathrm{cod}}\right)_{\delta \mathrm{p} / \mathrm{p}=0}($ at $\mathrm{H} 10)$ is shown in Table 1.

Record the values of $\left(\mathrm{X}_{\mathrm{cod}}\right)_{\delta \mathrm{p} / \mathrm{p}=0}($ at $\mathrm{H} 10)$ and $\left(\mathrm{X}_{\mathrm{cod}}^{\prime}\right)_{\delta \mathrm{p} / \mathrm{p}=0}($ at $\mathrm{H} 10)$ midway of the H10 septum magnet.

Table 1: Central Orbit Displacements at H10_septum.

\begin{tabular}{|c|c|c|}
\hline & $\begin{array}{c}\left(\mathrm{X}_{\text {cod }}\right) \delta \mathrm{p} / \mathrm{p}=0 \text { Bump Only } \\
\text { "Closed orbit" }[\mathrm{mm}]\end{array}$ & $\begin{array}{c}\left(\mathrm{X}_{\mathrm{cod}}\right)_{\delta \mathrm{p} / \mathrm{p}=0} \text { Bump }+ \text { G10kick } \\
\text { "Extracted orbit" }[\mathrm{mm}]\end{array}$ \\
\hline UpStream H10 & 51.5 & 83.5 \\
\hline Midway of H10 & 48.0 & 77.5 \\
\hline
\end{tabular}

2.2 The MAD code can now provide the 6x6 R_matrix of the FEB_section. We actually use the beam parameters at the beginning and end of the FEB section to calculate the matrix elements of the $\mathrm{R}$ matrix. This method includes the effect of the sextupoles on the beam which is laterally displaced at the location of the sextupoles.

2.3 Repeat task 2.1 but this time the central orbit should have increased momentum $\delta \mathrm{p} / \mathrm{p}=0.001$ and the starting coordinates at the middle of G10_kicker will be the coordinates $\left(\mathrm{X}_{\mathrm{cod}}\right)_{\mathrm{dp} / \mathrm{p}=0.001}$ (at G10) and $\left(\mathrm{X}_{\mathrm{cod}}^{\prime}\right)_{\mathrm{dp} / \mathrm{p}=0.001}$ (at G10) which were recorded from section (1.4 ii above) . Record the output coordinates $\left(\mathrm{X}_{\mathrm{cod}}\right)_{\mathrm{dp} / \mathrm{p}=0.001}\left(\right.$ at H10) and $\left(\mathrm{X}_{\mathrm{cod}}^{\prime}\right)_{\mathrm{dp} / \mathrm{p}=0.001}$ (at H10). These coordinates will help determine the dispersion function $\eta_{\mathrm{x}, \mathrm{y}}$ at the starting point of AtR line. 
In the next subsection we describe the procedure to calculate the R_matices of the FEB section using the "Raytrace" method.

\section{Calculation of the "FEB" matrices using the "Raytrace" method}

The procedure of calculating the R_matrices with the "Raytrace" method is similar to the method of using MAD, with two major differences:

a) It uses the "BEAM_model" [4] of the AGS ring, and

b) The AGS main magnets are described with the experimentally measured field maps [2].

Both features (a) and (b) above have been combined, in a modified version of the BEAM code[4]. A description, on how, the experimentally field maps of the main magnets, have been incorporated into the BEAM code, is given in Ref. [1].

\section{Procedure to calculate the $R$ _matrices of the FEB section using the "Raytrace" method}

In this section we describe the step by step procedure to calculate the R_matrices of the FEB section by using the modified BEAM code for the AGS ring.

1. Set up AGS for beam extraction using the "BEAM model" of the AGS :

1.1 Main magnets of AGS are set to allow a beam with the required "momentum at extraction" to circulate. The required "momentum at extraction" corresponds to the momentum of the beam which allows the beam to circulate at a radius of $\mathrm{R}_{0}=0.0[\mathrm{~mm}]$. The actual extraction momentum of the beam is achieved when the beam is moved to an outer radius and with the local beam extraction bumps "G10" and "H10" both energized.

1.2 The Tune_Quadrupoles and Chromaticity_sectupoles are set to generate the required tunes $\mathrm{Q}_{\mathrm{x}, \mathrm{y}}$ and chromaticities $\xi_{\mathrm{x}, \mathrm{y}}$.

1.3 The beam orbit is placed at the desired radius, with the local beam bumps for fast extraction set to provide the required $\mathrm{X}_{\text {cod }}$ at the middle of the G10_kicker and H10_septum [1,3] for the closed orbit. The required closed orbit displacements are: $\left(\mathrm{X}_{\mathrm{cod}}\right)_{\delta \mathrm{p} / \mathrm{p}=0}($ at G10)= 61 $[\mathrm{mm}]$ and $\left(\mathrm{X}_{\mathrm{cod}}\right)_{\delta \mathrm{p} / \mathrm{p}=0}($ at $\mathrm{H} 10)=\sim 48[\mathrm{~mm}]$.

1.4 The following physical quantities, of the closed beam orbit, are recorded:

i. The beam parameters $\left(\beta_{x y}, \alpha_{x y}\right)$ at the location of the G10_kicker.

ii. The $X_{\text {cod }}$ and $X_{\text {cod }}^{\prime}$ at the location of the G10 kicker for two different momenta of the central orbit, $\delta \mathrm{p} / \mathrm{p}=0$ and $\delta \mathrm{p} / \mathrm{p}=0.001$. It is required that $\left(\mathrm{X}_{\mathrm{cod}}\right)_{\delta \mathrm{p} / \mathrm{p}=0}($ at $\mathrm{G} 10)=\sim 61[\mathrm{~mm}]$ and $\left(\mathrm{X}_{\mathrm{cod}}\right) \delta \mathrm{p} / \mathrm{p}=0$ (at $\mathrm{H} 10)=\sim 48[\mathrm{~mm}]$ (Distance is measured from the Optimum Closed Orbit (OCO) ).

2. After the orbit is closed, we use the BEAM model of the section of the AGS between the middle of the G10_kicker and the middle of the H10_septum and we perform the following operations. 
2.1 With the magnetic elements of the FEB section set as in item (1.) above, and the beam's initial conditions same as those recorded in (1.5), we kick the beam with the G10_kicker. The strength of the G10_kicker should be such that the central ray of the beam, which is referred as, "Extracted orbit" in Table 1., should have the required displacement $\left(\mathrm{X}_{\mathrm{cod}}\right)_{\delta \mathrm{p} / \mathrm{p}=0}$ at the center of the H10_septum. This

$\left(\mathrm{X}_{\mathrm{cod}}\right)_{\delta \mathrm{p} / \mathrm{p}=0}$ displacement is shown in Table 1 .

Record the values of $\left(\mathrm{X}_{\mathrm{cod}}\right)_{\delta \mathrm{p} / \mathrm{p}=0}($ at $\mathrm{H} 10)$ and $\left(\mathrm{X}_{\mathrm{cod}}^{\prime}\right)_{\delta \mathrm{p} / \mathrm{p}=0}($ at $\mathrm{H} 10)$ The BEAM code can now provide the Horizontal and Vertical 2x2 $\mathrm{R}$ _matrices of the FEB_section. The $\mathrm{R}_{16}$ and $\mathrm{R}_{26}$ matrix elements can also be computed.

2.2 Repeat task 2.1 but this time the central orbit should have an increased momentum of $\mathrm{dp} / \mathrm{p}=0.001$ and the starting coordinates will be the coordinates $\left(\mathrm{X}_{\mathrm{cod}}\right)_{\mathrm{dp} / \mathrm{p}=0.001}($ at $\mathrm{G} 10)$ and $\left(\mathrm{X}_{\mathrm{cod}}^{\prime}\right)_{\mathrm{dp} / \mathrm{p}=0.001}$ (at G10) which were recorded from section (1.4 ii ). Record the output coordinates $\left(\mathrm{X}_{\mathrm{cod}}\right)_{\mathrm{dp} / \mathrm{p}=0.001}\left(\right.$ at H10) and $\left(\mathrm{X}_{\mathrm{cod}}^{\prime}\right)_{\mathrm{dp} / \mathrm{p}=0.001}($ at H10). These coordinates will help determine the dispersion function $\eta_{\mathrm{x}, \mathrm{y}}$ at the starting point of AtR line.

In the next subsection we describe the procedure to calculate the R_matices of the DEC section using the "Raytracing" method.

\section{Calculation of the "DEC" matrices using the "Raytracing" method}

Although the DEC section does not include any lumped magnetic elements like dipoles, quadrupoles etc., it cannot be considered as a drift space because it is located in the fringe field region of the H11, H12 and H13 AGS main magnets which generate a field which is described by the magnetic field maps of the main magnets [2]. For this section of the line we use the "Raytrace method" to calculate the R_matrices.

\section{Procedure to calculate the $R \_$matrices of the DEC section using the Raytrace method}

The Raytrace method is currently the only method used to calculate the R_matrices of the DEC section. The rest of this subsection is devoted in the procedure used to calculate the R_matrices in the DEC section.

In the earlier subsections which describe the procedure to calculate the R_matrices for the FEB section, we energized the G10_kicker to kick the central orbit of the beam, for the central orbit to acquire a displacement at the middle of the H10 straight section $\mathrm{X}_{\text {cod }}($ at $\mathrm{H} 10)=\sim 77.5[\mathrm{~mm}]$.

Now using the modified BEAM code or the modified RAYTRACE code which both have incorporated the measured field maps of the main AGS magnet as well as the field maps of the fringe fields, we excite the H10_septum to bend the central orbit of the beam outside of the AGS, and achieve a central orbit displacement at the middle of the H13 straight section of $X_{\text {cod }}($ at H13) $=\sim 450.5[\mathrm{~mm}]$. This point which is at a distance of 450.5 [mm] from the middle of the straight section (H13), is defined as the starting point of the AtR line. The direction of the central orbit at the beginning of the AtR line should 
be $\mathrm{X}_{\text {cod }}^{\prime}($ at $\mathrm{H} 13)=\sim 69.5$ [mrad]. If the direction of the beam at the middle of the straight section H13 differs by more than \pm 1.0 mrad from the specified direction of $69.5 \mathrm{mrad}$, we should revisit the calculations of the FEB section, and if needed, we can change the strength of the G10_kicker. The possibility that the beam coordinates at H13 are not equal to $\left\{X, X^{\prime}\right\}=\{450.5[\mathrm{~mm}], 69.5[\mathrm{mrad}]\}$ when the beam position $\mathrm{X}$, at $\mathrm{H} 10$ is 75 [mm] will indicate that the fringe fields generated by H11,H12 and H13 main magnets do not scale linearly with magnet current (beam momentum). The fringe field linearity however has been tested by raytracing in this fringe field region, beams of two different momenta of $24.3 \mathrm{GeV} / \mathrm{c}$ and $29.0 \mathrm{GeV} / \mathrm{c}$.

With the central orbit extracted from the AGS and having the required trajectory in the DEC section, the modified BEAM or RAYTRACE codes can be used to calculate the R_matix in the DEC section.

\section{Calculation of the beam parameters $\left(\alpha_{x, y}, \beta_{x, y}\right)$ and $\left(\eta_{x, y}, \eta^{\prime}{ }_{x, y}\right)$ at $\mathbf{H 1 3}$}

With the central orbit extracted from the AGS and having the required trajectory in the DEC section, the modified BEAM or RAYTRACE codes can be used to calculate the R_matix in the DEC section.

With the R_matrices calculated, as described in the previous sections, the beam parameters at the starting point of the AtR line can be computed by using the relations:

$$
\begin{aligned}
& \beta_{\mathrm{x}}(\mathrm{H} 13)=\left(\mathrm{R}_{11} \mathrm{R}_{11}\right) \beta_{\mathrm{x}}(\mathrm{H} 10)-2 \mathrm{R}_{11} \mathrm{R}_{12} \alpha_{\mathrm{x}}(\mathrm{H} 10)+\left(\mathrm{R}_{12} \mathrm{R}_{12}\right) \gamma_{\mathrm{x}}(\mathrm{H} 10) \\
& \alpha_{\mathrm{x}}(\mathrm{H} 13)=-\left(\mathrm{R}_{11} \mathrm{R}_{21}\right) \beta_{\mathrm{x}}(\mathrm{H} 10)+\left(1+2 \mathrm{R}_{11} \mathrm{R}_{21}\right) \alpha_{\mathrm{x}}(\mathrm{H} 10)-\left(\mathrm{R}_{12} \mathrm{R}_{22}\right) \gamma_{\mathrm{x}}(\mathrm{H} 10) \\
& \beta_{\mathrm{y}}(\mathrm{H} 13)=\left(\mathrm{R}_{33} \mathrm{R}_{33}\right) \beta_{\mathrm{y}}(\mathrm{H} 10)-2 \mathrm{R}_{33} \mathrm{R}_{34} \alpha_{\mathrm{y}}(\mathrm{H} 10)+\left(\mathrm{R}_{34} \mathrm{R}_{34}\right) \gamma_{\mathrm{y}}(\mathrm{H} 10) \\
& \alpha_{\mathrm{y}}(\mathrm{H} 13)=-\left(\mathrm{R}_{33} \mathrm{R}_{43}\right) \beta_{\mathrm{y}}(\mathrm{H} 10)+\left(1+2 \mathrm{R}_{33} \mathrm{R}_{43}\right) \alpha_{\mathrm{y}}(\mathrm{H} 10)-\left(\mathrm{R}_{34} \mathrm{R}_{44}\right) \gamma_{\mathrm{y}}(\mathrm{H} 10)
\end{aligned}
$$

The $\mathrm{R}_{\mathrm{ij}}$ matrix elements appearing in the relations above correspond to the matrix elements of the line between the middle of the H10_septum and the starting point of the AtR line (H13 point). The dispersion functions $\eta_{x}(\mathrm{H} 13)$ and $\eta^{\prime}{ }_{x}(\mathrm{H} 13)$ are calculated using the following relations:

$$
\begin{aligned}
& \eta_{\mathrm{x}}(\mathrm{H} 13)=\left(\mathrm{X}_{\mathrm{cod}}(\mathrm{H} 13)_{\delta \mathrm{p} / \mathrm{p}=0.001}-\mathrm{X}_{\mathrm{cod}}(\mathrm{H} 13)_{\delta \mathrm{p} / \mathrm{p}=0.000}\right) / 0.001 \\
& \eta^{\prime}{ }_{\mathrm{x}}(\mathrm{H} 13)=\left(\mathrm{X}_{\mathrm{cod}}(\mathrm{H} 13)_{\delta \mathrm{p} / \mathrm{p}=0.001}-\mathrm{X}_{\mathrm{cod}}(\mathrm{H} 13)_{\delta \mathrm{p} / \mathrm{p}=0.000}\right) / 0.001
\end{aligned}
$$

The quantities $\mathrm{X}_{\mathrm{cod}}(\mathrm{H} 13)_{\delta \mathrm{p} / \mathrm{p}=0.000}$ and $\mathrm{X}_{\mathrm{cod}}^{\prime}(\mathrm{H} 13)_{\delta \mathrm{p} / \mathrm{p}=0.000}$ are the displacement and direction respectively, of the central orbit which starts from the middle of the straight section $\mathrm{H} 10$ with specified coordinates $\mathrm{X}_{\mathrm{cod}}(\mathrm{H} 10)_{\delta \mathrm{p} / \mathrm{p}=0.000}$ and $\mathrm{X}_{\operatorname{cod}}(\mathrm{H} 13)_{\delta \mathrm{p} / \mathrm{p}=0.000}$ and ends up at the middle of the straight section $\mathrm{H} 13$ after passing through the fringe field of the H11, H12 and H13 AGS main magnets.

The quantities $\mathrm{X}_{\text {cod }}(\mathrm{H} 13)_{\delta \mathrm{p} / \mathrm{p}=0.001}$ and $\mathrm{X}_{\mathrm{cod}}^{\prime}(\mathrm{H} 13)_{\delta \mathrm{p} / \mathrm{p}=0.001}$ are the displacement and direction respectively, of the central orbit which starts from the middle of the straight section $\mathrm{H} 10$ with specified coordinates $\mathrm{X}_{\mathrm{cod}}(\mathrm{H} 10)_{\delta \mathrm{p} / \mathrm{p}=0.001}$ and $\mathrm{X}_{\text {cod }}(\mathrm{H} 13)_{\delta \mathrm{p} / \mathrm{p}=0.001}$ and end up at the middle of the straight section $\mathrm{H} 13$ after passing through the fringe field of the H11, H12 and H13 AGS main magnets. 


\section{Results}

In this section we will present the results from the calculations.

The results are summarizes in the Table 2 below. The first six columns of the Table 1 correspond to the extraction setting of the AGS synchrotron.

a) column 1: The rigidity of the central momentum of the circulating beam at $\mathrm{R}=0.0$ $[\mathrm{mm}]$. This rigidity corresponds to the setting of the AGS main magnets during extrtaction.

b) column 2: Average Extraction radius of the circulating beam just before extraction.

The local beam bumps at the middle of the G10_kicker and the middle of the H10_septum are set to provide $\left(\mathrm{X}_{\mathrm{cod}}\right)_{\delta \mathrm{p} / \mathrm{p}=0}($ at $\mathrm{G} 10)=\sim 61[\mathrm{~mm}]$ and $\left(\mathrm{X}_{\mathrm{cod}}\right)_{\delta \mathrm{p} / \mathrm{p}=0}$ (at $\mathrm{H} 10)=\sim 48[\mathrm{~mm}]$ independent of the average beam radius.

c) columns 3,4: Tune Quadrupoles set for specific tunes.

d) columns 5,6: Chromaticity sextupoles set for specific chromaticities.

e) column 7: The name of the Table_Xi which contains results corresponding to the extraction settings of the AGS as shown in the first six columns of Table 1. The

Tables_Xi contain information like the beam parameters at the beginning of the AtR line an the R_matrices of the "FEB" and "DEC" sections.

f) column 8: The column under the name "comments" helps the reader to group together the Tables_Xi in order to find how the beam parameters at the beginning of the AtR line, vary as the extraction setting of the AGS vary.

Table 2: This Table provides the names of the Tables (column 7) generated for the various extraction settings of the AGS

\begin{tabular}{|c|c|c|c|c|c|c|c|}
\hline $\begin{array}{c}\text { Brho[ } \\
\text { T.m] }\end{array}$ & $\begin{array}{c}\mathrm{R}_{0} \\
{[\mathrm{~mm}} \\
]\end{array}$ & $\begin{array}{c}\mathrm{IQx} \\
{[\mathrm{A}]}\end{array}$ & $\begin{array}{c}\text { IQy } \\
{[\mathrm{A}]}\end{array}$ & $\begin{array}{c}\text { ISx } \\
{[\mathrm{A}]}\end{array}$ & $\begin{array}{c}\text { ISy } \\
{[\mathrm{A}]}\end{array}$ & Table & Comments \\
\hline 81.055 & -2.1 & 0.0 & 0.0 & 0.0 & 0.0 & X1 & $\begin{array}{c}\text { Bare_AGS } \\
\text { VaryExtraction Radius }\end{array}$ \\
\hline 81.055 & -4.7 & 0.0 & 0.0 & 0.0 & 0.0 & $\mathrm{X} 2$ & $\begin{array}{c}\text { Bare_AGS } \\
\text { VaryExtraction Radius }\end{array}$ \\
& & 8.621 & 8.748 & -24.1 & 4.7 & & Bare_AGS \\
\hline 81.055 & -7.3 & 0.0 & 0.0 & 0.0 & 0.0 & $\mathrm{X} 3$ & VaryExtraction Radius \\
\hline 81.055 & -2.1 & 339 & -130 & 104 & 80 & X4 & No Bare AGS \\
& & 8.757 & 8.751 & -28.4 & 4.6 & & X4 compare X5 \\
\hline 81.055 & -4.7 & 339 & -130 & 104 & 80 & X5 & No Bare AGS \\
& & 8.718 & 8.768 & -28.4 & 4.6 & & X4 compare X5 \\
\hline 88.233 & -4.89 & 330 & -210 & 120 & 100 & X6 & Au 2008 Extraction \\
& & 8.584 & 8.689 & -36.1 & 7.0 & & D 2008 Extraction \\
\hline 68.570 & -4.7 & 120 & -90 & 80 & 60 & X7 & D \\
& & 8.629 & 8.689 & -27.3 & 1.8 & & \\
\hline
\end{tabular}


Each Table_Xi is included in this section below, and the explanation of each physical quantity included in each Table_Xi is given below.

Row 1: Designates the computer code used in the calculations, (MAD) or (BEAM)

Row 2: The central momentum of the circulating beam in $\mathrm{GeV} / \mathrm{c}$ at $\mathrm{R}=0[\mathrm{~mm}]$. This corresponds to specified AGS main magnets setting which is always the same during the extraction process.

Row 3: The amount of the momentum increase added to the momentum of the beam shown in Row 2. This momentum increase takes the circulating beam to the specified radius at beam extraction.

Row 4: The radius of the circulating beam. The reason that the radius of the circulating beam for $\mathrm{dp} / \mathrm{p}=0$ is $\mathrm{R}$ ? $0[\mathrm{~mm}]$ in both the MAD and BEAM calculations, instead of $\mathrm{R}=0[\mathrm{~mm}]$ is due to the increase of the beam path of the circulating beam when the extraction local beam bumps are energized.

Row5 to 8: The settings of the tune quadrupoles and chromaticity sextupoles in [A]

Row 9, 10: The strength of the backleg winding of the G10 and H10 local beam bumps. Each of the local bump (G10 or H10) is generated by a set of backleg windings placed at specific main magnets and connected in series. G10 or H10 is energized by a single power supply. For more details about the configuration of the G10 and H10 local beam bumps see Ref $[1,3]$

Rows 11,12: The values of the $\mathrm{X}_{\mathrm{cod}}(\mathrm{G} 10)$ and $\mathrm{X}_{\mathrm{cod}}^{\prime}(\mathrm{G} 10)$ of the circulating beam midway of the G10_kicker.

Rows 13,14: The values of the horizontal and vertical beam parameters of the circulating beam midway of the G10_kicker.

Rows 15, 20: Same as the corresponding rows 9 through 14 but for middle of the H10_septum

Rows 21,24: The Horizontal and Vertical Tunes and chomaticities during the circulating beam, before extraction.

Row 25: The strength of the G10_kicker required to place the $\mathrm{X}_{\mathrm{cod}}$ extracted beam at $\mathrm{H} 10$ at 77.5 [mm].

Rows 26,27: The values of the $\mathrm{X}_{\mathrm{cod}}(\mathrm{H} 10)$ and $\mathrm{X}_{\text {cod }}^{\prime}(\mathrm{H} 10)$ of the extracted beam at the middle of the H10_septum.

Rows 28,29: The values of the horizontal and vertical beam parameters of the extracted beam at the middle of the H10_septum.

Row 30: The value of the horizontal phase advance in units of $2 \pi$ from the middle of the G10_kicker to the middle of the H10_septum.

Rows 31,33: Same as in rows 28 to 30 but for the vertical.

The rest of the Tables_Xi show:

a) the R_matrices of the FEB_section, which is defined from the middle of the G10_kicker to the middle of the H10_septum,

b) the R_matrices of the DEC_section, which is defined from the middle of the H10_septum to the beginning of the AtR line. 
c) the beam coordinates of the extracted beam and the parameters at the beginning of the AtR line.

\section{Comments on comparing the various Tables_Xi}

1. The tables $\mathrm{X} 1, \mathrm{X} 2, \mathrm{X} 3$ can be used to obtain information on the R_matrices and on how the beam parameters at the beginning of the AtR line vary as a function of extraction radius. (Beam rigidity $=81.055$ [T.m] and Bare AGS )

2. The tables X4 and X5 provide the same information as item 1 above but for for Non Bare AGS. The tune quadrupoles and the chromaticity Sextupoles of the BEAM code were set to provide same tunes and chromaticities as in MAD code.

3. The tables $\mathrm{X} 6$ and $\mathrm{X} 7$ provide the same information on the extraction beam parameters for the Au2008 and D2008 run. The same set currents were used for the tune quadrupoles and chromaticity sextupoles of the MAD and BEAM codes. Therefore the tunes and chromaticities as derived from the MAD code are not equal to those as derived from the BEAM code. No measured tunes and chromaticities for the Au2008 and D2008 run at this time. The fact that MAD code and the BEAM code do not provide the same tunes and chromaticities under the same settings of the AGS is a major issue under investigation.

\section{Further development of the "AtR BPM Application"}

In this section we propose two methods to further automate the "AtR BPM Application"

1. The calculations of a number of R_matrices and of extraction beam parameters as a function of the extraction setup of the AGS can generate a data base of R_matrices and beam parameters which can be called once the extraction settings of the AGS are known. Subsequently an optimization will follow to properly match the beam from the U_line to the $\mathrm{W}_{-}$line and also to satisfy the beam constraints of the U_line. This optimization will set the values of the various quadrupoles of the U_line.

2. An alternative method of automating the extraction setup as well as the "AtR BPM Application" is to read into a code the AGS settings at extraction and automate the procedure we describe in this note of how to calculate the R_matrices and the beam parameters at the beginning of the AtR line. Subsequently an optimization will follow to properly match the beam from the U_line to the W_line. This optimization will set the values of the various quadrupoles of the U_line.

\section{References}

[1] N. Tsoupas, et. al. "Closed Orbit Calculations at AGS and Extraction Beam Parameters at H13" AD/RHIC/RD-75 
[2] R. Thern, Provided the experimentally measured field maps for the AGS main magnets. A description of the field maps is provided in Ref. [1].

[3] N. Tsoupas, et. al. "Fast Extracted Beam (FEB) for the g-2 Experiment" $\mathrm{CA} / \mathrm{AP} / 54$

[4] G. H. Morgan, "Fortran IV Version of 'BEAM' the AGS Orbit Computing Program" AGS Internal Repot.

C. J. Gardner, Private Communication.

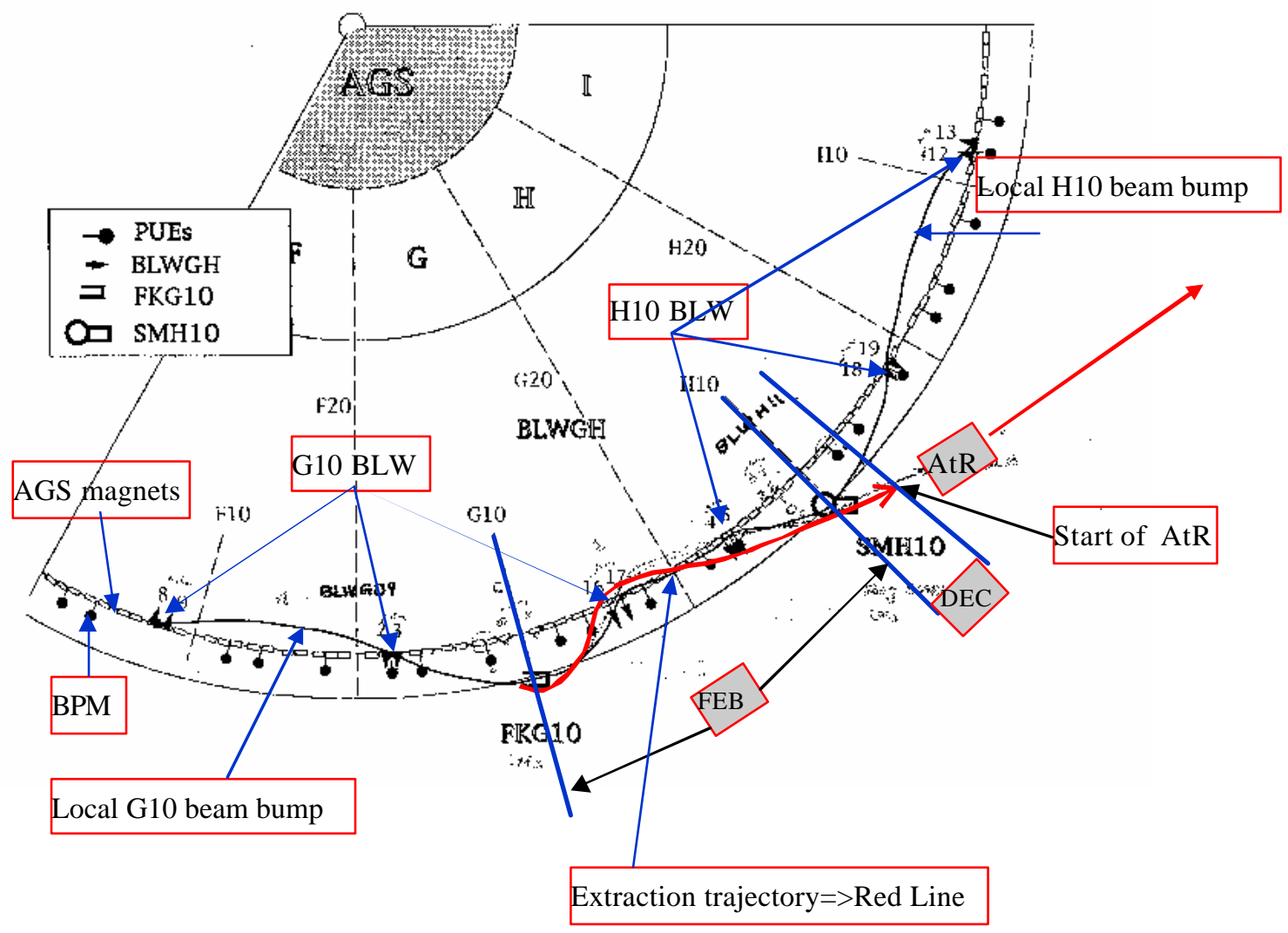

Figure 1: Schematic diagram of the section of the AGS ring which includes the Fast Extracted Beam region. The very small boxes located along an arc are the AGS main magnets. The black dots which are close to the AGS magnets are the Beam Position Monitors BPM's of the AGS ring. The G10_kicker is the "FKG10" and the H10_septum is the "SMH10". The Back Leg Windings for the G10 and H10 orbit bumps The black solid line designates the circulating beam just before extraction with the local bumps activated. The red line is the extracted beam trajectory which continues down the AtR line. The span of the "FEB" beam line 
sections and of the "DEC" line section which are discussed in the text, are shown in the figure.

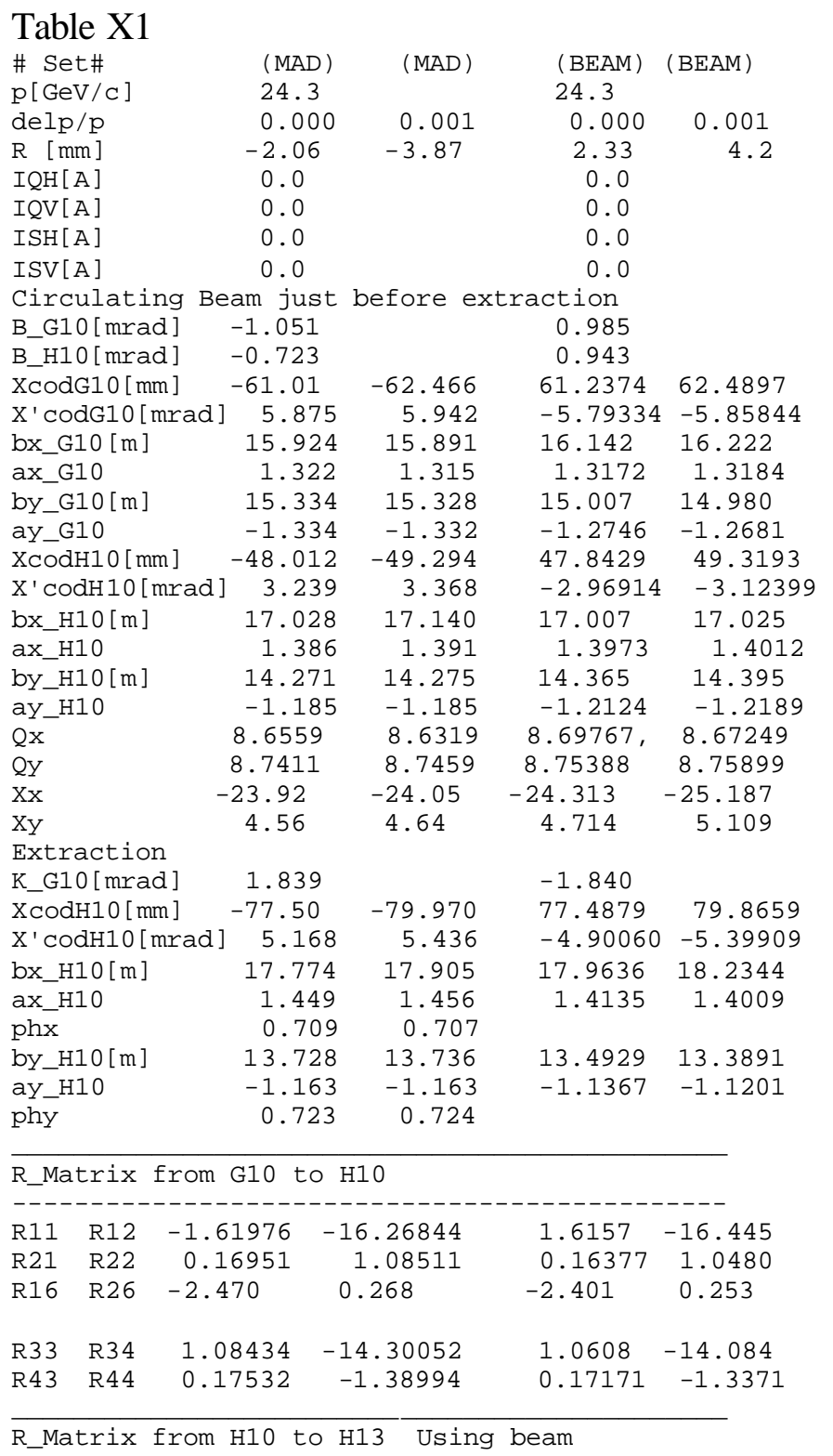




$\begin{array}{llll}\text { R11 } & \text { R12 } & 2.2899 & 14.527 \\ \text { R21 } & \text { R22 } & 0.26409 & 2.1120 \\ \text { R16 } & \text { R26 } & 0.1521 & 0.0149 \\ & & & \\ \text { R33 } & \text { R34 } & 0.050164 & 6.2662 \\ \text { R43 } & \text { R44 } & -0.15799 & 0.19918\end{array}$

Beam Parameters at H13 Using Beam

$\begin{array}{ll}\mathrm{XcodH} 13[\mathrm{~mm}] & 450.6762\end{array}$

X' codH10 [mrad] $\quad 69.374$

bx_H13 [m] $\quad 35.3760$

ax_H13 $\quad-3.7250$

ex $[\mathrm{m}] \quad-1.398$

$\begin{array}{ll}\mathrm{e}^{\prime} \mathrm{x} & -0.1077\end{array}$

by_H13[m] 7.4187

ay_H13 1.0089

\section{Table X2}

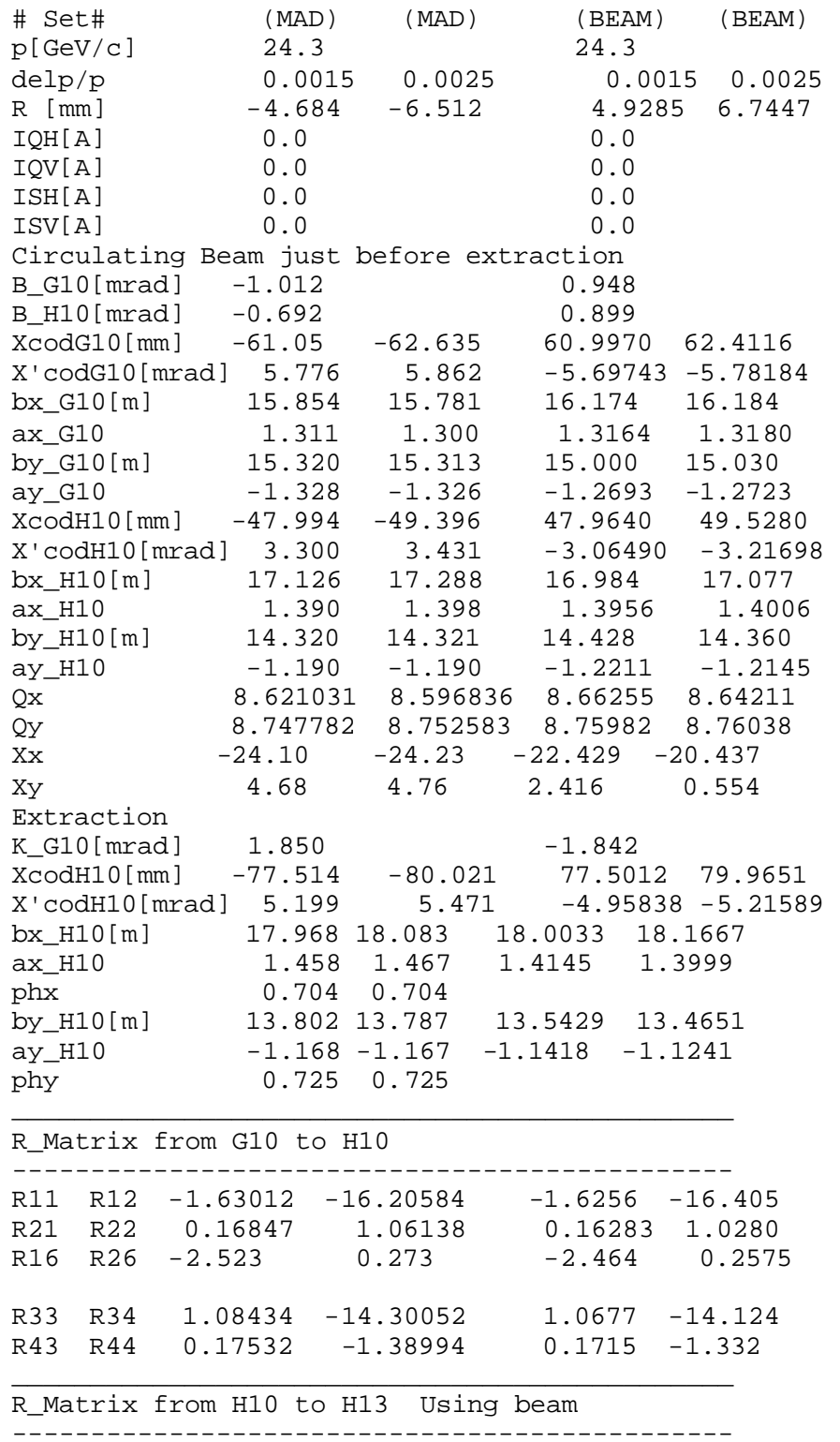




$\begin{array}{llll}\text { R11 } & \text { R12 } & 2.2880 & 14.520 \\ \text { R21 } & \text { R22 } & 0.26367 & 2.110 \\ \text { R16 } & \text { R26 } & 0.1528 & 0.0150 \\ & & & \\ \text { R33 } & \text { R34 } & 0.051236 & 6.2703 \\ \text { R43 } & \text { R44 } & -0.15785 & 0.20001\end{array}$

Beam Parameters at 113 Using Beam

$\begin{array}{lc}\text { XcodH13 [mm] } & 450.6532 \\ X^{\prime} \text { codH10 [mrad] } & 69.37962 \\ \text { bx_H13 [m] } & 35.4013 \\ \text { ax_H13 } & -3.7231 \\ \text { ex[m] } & -1.646 \\ \text { e'x } & -0.1362 \\ \text { by_H13 [m] } & 7.4582 \\ \text { ay_H13 } & 1.0146\end{array}$

Table X3

\begin{tabular}{|c|c|c|c|c|c|c|}
\hline \multicolumn{3}{|c|}{ \# Set\# } & \multicolumn{2}{|c|}{1 (MAD) 1 (MAD) } & \multicolumn{2}{|c|}{1 (BEAM) 1 (BEAM) } \\
\hline \multicolumn{3}{|c|}{$\mathrm{p}[\mathrm{GeV} / \mathrm{c}]$} & 24.3 & & 24.3 & \\
\hline \multicolumn{3}{|c|}{$\operatorname{delp} / \mathrm{p}$} & 0.0030 & 0.0040 & 0.0030 & 0.0040 \\
\hline \multicolumn{3}{|c|}{$R \quad[\mathrm{~mm}]$} & -7.3361 & -9.1826 & 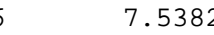 & 9.3676 \\
\hline \multicolumn{3}{|c|}{$\mathrm{IQH}[\mathrm{A}]$} & 0.0 & & 0.0 & \\
\hline \multicolumn{3}{|c|}{$\mathrm{IQV}[\mathrm{A}]$} & 0.0 & & 0.0 & \\
\hline \multicolumn{3}{|c|}{ ISH [A] } & 0.0 & & 0.0 & \\
\hline \multicolumn{3}{|c|}{ ISV $[\mathrm{A}]$} & 0.0 & & 0.0 & \\
\hline \multicolumn{7}{|c|}{ Circulating Beam just before extraction } \\
\hline \multicolumn{4}{|c|}{ B_G10 [mrad] $\quad-0.970$} & & 0.912 & \\
\hline \multicolumn{3}{|c|}{ B_H10 [mrad $]$} & -0.660 & & 0.846 & \\
\hline \multicolumn{3}{|c|}{$\mathrm{XcodG10}[\mathrm{mm}]$} & -61.108 & -62.804 & 61.0863 & 62.6244 \\
\hline \multicolumn{3}{|c|}{$X^{\prime} \operatorname{codG} 10$ [mrad] } & ] 5.689 & 5.791 & 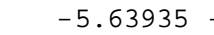 & -5.73939 \\
\hline \multicolumn{3}{|c|}{ bx_G10 [m] } & 15.723 & 15.537 & 16.187 & 16.202 \\
\hline \multicolumn{3}{|c|}{ ax_G10 } & 1.294 & 1.271 & 1.3183 & 1.3166 \\
\hline \multicolumn{3}{|c|}{ by_G10 [m] } & 15.304 & 15.296 & 15.038 & 15.032 \\
\hline \multicolumn{3}{|c|}{ ay_G10 } & -1.322 & -1.320 & -1.2727 & -1.2707 \\
\hline \multicolumn{3}{|c|}{$\mathrm{XcO} d \mathrm{dH} 10[\mathrm{~mm}]$} & -48.102 & -49.610 & 47.7821 & 49.4283 \\
\hline \multicolumn{3}{|c|}{$\mathrm{X}^{\prime} \mathrm{codH} 10$ [mrad] } & 3.359 & 3.493 & -3.12865 & -3.27965 \\
\hline \multicolumn{3}{|c|}{$\mathrm{bx} \_\mathrm{H} 10[\mathrm{~m}]$} & 17.298 & 17.596 & 17.030 & 17.109 \\
\hline \multicolumn{3}{|c|}{ ax_H10 } & 1.399 & 1.416 & 1.3970 & 1.3998 \\
\hline by_- & $10[\mathrm{~m}]$ & & 14.366 & 14.364 & 14.389 & 14.378 \\
\hline ay_- & & & -1.195 & -1.194 & -1.2164 & -1.2154 \\
\hline Qx & & & 8.585995 & 8.56164 & 8.63386 & 8.61009 \\
\hline Qy & & & 8.754538 & 8.75944 & 8.75977 & 8.76388 \\
\hline $\mathrm{Xx}$ & & & -24.29 & -24.43 & -21.986 & 23.776 \\
\hline $\mathrm{xy}$ & & & 4.79 & 4.89 & 2.229 & 4.104 \\
\hline Ext & actio & & & & & \\
\hline K_G & $0[\mathrm{mra}$ & & 1.853 & & -1.842 & \\
\hline $\mathrm{XcO}$ & $\mathrm{H} 10[\mathrm{~m}$ & & -77.501 & -80.075 & 77.5012 & 79.9651 \\
\hline$X^{\prime} C$ & $\mathrm{dH} 10[$ & mrad] & ] 5.219 & 5.497 & -4.95838 & -5.21589 \\
\hline $\mathrm{bx}$ & $10[\mathrm{~m}]$ & & 18.099 & 18.167 & 18.0033 & 1667 \\
\hline$a x_{-}$ & & & 1.468 & 1.469 & 1.4145 & 3999 \\
\hline $\operatorname{phx}$ & & & 0.704 & 0.702 & & \\
\hline by_. & $10[\mathrm{~m}]$ & & 13.833 & 13.853 & 13.5429 & 4651 \\
\hline ay_- & & & -1.172 & -1.167 & -1.1418 & 1241 \\
\hline phy & & & 0.725 & 0.726 & & \\
\hline$R \_M$ & trix & from & G10 to $\mathrm{H}$ & $\mathrm{H} 10$ & & \\
\hline & $\mathrm{R} 12$ & -1.6 & 63655 & -16.16952 & -1.6329 & -16.354 \\
\hline R21 & R22 & 0.1 & 16770 & 1.04585 & 0.16175 & 1.0075 \\
\hline R16 & R2 6 & -2.5 & 574 & 0.278 & -2.517 & 0.2613 \\
\hline R33 & R34 & 1.0 & 09266 & -14.37079 & 1.0730 & -14.165 \\
\hline R43 & R44 & 0.1 & 17467 & -1.38211 & 0.17114 & -1.3272 \\
\hline$R \_M$ & $\operatorname{trix}$ & from & H10 to $\mathrm{F}$ & H13 Using & $\mathrm{be}$ & \\
\hline R11 & $\mathrm{R} 12$ & & & & 2.2870 & .518 \\
\hline R21 & R22 & & & & 0.263592 . & .111 \\
\hline
\end{tabular}




$\begin{array}{llcc}\text { R16 } & \text { R26 } & 0.1528 & 0.0149 \\ \text { R33 } & \text { R34 } & 0.051925 & 6.2716 \\ \text { R43 } & \text { R44 } & -0.15779 & 0.1999\end{array}$

Beam Parameters at H13 Using Beam

$\begin{array}{lc}\text { XcodH13 [mm] } & 450.2426 \\ \text { X'codH10 [mrad] } & 69.32804 \\ \text { bx_H13 [m] } & 35.5953 \\ \text { ax_H13 } & -3.7471 \\ \text { ex[m] } & -1.860 \\ \text { e'x } & -0.161 \\ \text { by_H13 [m] } & 7.4314 \\ \text { ay_H13 } & 1.0145\end{array}$

\section{Table X4}

\begin{tabular}{|c|c|c|c|c|}
\hline \# Set\# & 1 (MAD & 1 (MAD) & 1 (BEAM) & 1 (BEAM) \\
\hline $\mathrm{p}[\mathrm{GeV} / \mathrm{c}]$ & 24.3 & & 24.3 & \\
\hline $\mathrm{delp} / \mathrm{p}$ & 0.000 & 0.001 & 0.000 & 0.001 \\
\hline$R \quad[\mathrm{~mm}]$ & -2.1 & -3.9 & 2.4 & 4.2 \\
\hline $\mathrm{IQH}[\mathrm{A}]$ & 339.0 & & 300.0 & \\
\hline$I Q V[A]$ & $-130 \cdot 0$ & & -200.0 & \\
\hline ISH $[\mathrm{A}]$ & 104.0 & & 100.0 & \\
\hline $\operatorname{ISV}[\mathrm{A}]$ & 80.0 & & 80.0 & \\
\hline G10 [mrad] & -0.998 & & 0.940 & \\
\hline B_H10 [mrad] & -0.677 & & 0.928 & \\
\hline $\mathrm{XcodG10}[\mathrm{mm}]$ & -61.03 & -61.538 & 61.1593 & 61.4625 \\
\hline$X^{\prime} \operatorname{codg} 10$ [mrad] & 6.189 & 6. & -5.96154 & -5 . \\
\hline $\mathrm{bx} \_\mathrm{G} 10[\mathrm{~m}]$ & 15.563 & 15.4 & 15.833 & 15. \\
\hline ax_G10 & 1.361 & 1.3 & 1.3469 & 1.3248 \\
\hline by_G10 [m] & 18.215 & 18.170 & 17.558 & 17.628 \\
\hline ay_G10 & -1.674 & -1.669 & -1.5604 & -1.5633 \\
\hline $\mathrm{XcodH} 10[\mathrm{~mm}]$ & -48.00 & -48.908 & 47.8522 & 49. \\
\hline$X^{\prime} \operatorname{codH} 10$ [mrad] & 3.260 & 3.405 & -3.01732 & -3.21570 \\
\hline $\mathrm{bx} \_\mathrm{H} 10[\mathrm{~m}]$ & 20.716 & 21.090 & 20.679 & 20.980 \\
\hline ax_H10 & 1.775 & 1.803 & 1.7779 & 1.8045 \\
\hline by_H10 [m] & 12.401 & 12.461 & 12.484 & 12.506 \\
\hline ay_H10 & -1.078 & -1.084 & -1.1186 & -1.1245 \\
\hline Qx & 8.757 & 8.728 & 8.77925 & 8.75164 \\
\hline Qy & 8.751 & 8.755847 & 8.77420 & 8.77852 \\
\hline $\mathrm{Xx}$ & $-28 \cdot 36$ & -28.618700 & -27.028 & -27.612 \\
\hline $\mathrm{xy}$ & 4.61 & 4.650659 & 4.138 & 4.318 \\
\hline \multicolumn{5}{|l|}{ Extraction } \\
\hline K_G10 [mrad] & 1.484 & & -1.720 & \\
\hline $\mathrm{XcodH} 10[\mathrm{~mm}]$ & -77.50 & -78.370 & 77.4617 & 79.8659 \\
\hline $\mathrm{X}^{\prime} \mathrm{codH} 10$ [mrad] & 5.45 & 5.563 & -5.15319 & -5.39909 \\
\hline $\mathrm{bx} \_\mathrm{H} 10[\mathrm{~m}]$ & 19.772 & 20.160 & 20.1222 & 20.6362 \\
\hline ax_H10 & 1.743 & 1.776 & 1.6931 & 1.7120 \\
\hline $\mathrm{ph} \bar{x}$ & 0.714 & 0.710 & & \\
\hline by_H10 [m] & 13.181 & 13.237 & 12.7951 & 12.7135 \\
\hline ay_H10 & -1.150 & -1.154 & -1.1241 & -1.1096 \\
\hline phy & 0.716 & 0.717 & & \\
\hline
\end{tabular}

\begin{tabular}{|c|c|c|c|}
\hline$R \_M c$ & $\operatorname{trix}$ & from G10 to H10 & \\
\hline R11 & $\mathrm{R} 12$ & $-1.75339-17.03986$ & $1.7551-17.263$ \\
\hline R21 & R22 & 0.19285 & $0.18268 \quad 1.227$ \\
\hline R16 & R2 6 & -2.427 & -2.404 \\
\hline R33 & R34 & $1.21130-15.14267$ & $1.1812-14.802$ \\
\hline R43 & R4 4 & $0.19166-1.57037$ & $0.18604-1.4847$ \\
\hline$R \_M$ & $x$ & com H10 to H13 & \\
\hline $\begin{array}{l}\text { R11 } \\
\text { R21 }\end{array}$ & $\begin{array}{l}\text { R12 } \\
\text { R22 }\end{array}$ & & $\begin{array}{ll}2.2903 & 14.528\end{array}$ \\
\hline
\end{tabular}




\begin{tabular}{llcl} 
R16 R26 & 0.156 & 0.0154 \\
R33 R34 & 0.050012 & 6.2652 \\
R43 R44 & -0.15802 & 0.1989 \\
& & \\
\hline Beam Parameters at H13 & \\
-------------- \\
bx_H13 [m] & 33.4364 \\
ax_H13 & -3.3830 \\
ex[m] & -0.722 \\
e'x & -0.013 \\
by_H13 [m] & 7.6818 \\
ay_H13 & 0.9825 \\
&
\end{tabular}

\section{Table X5}

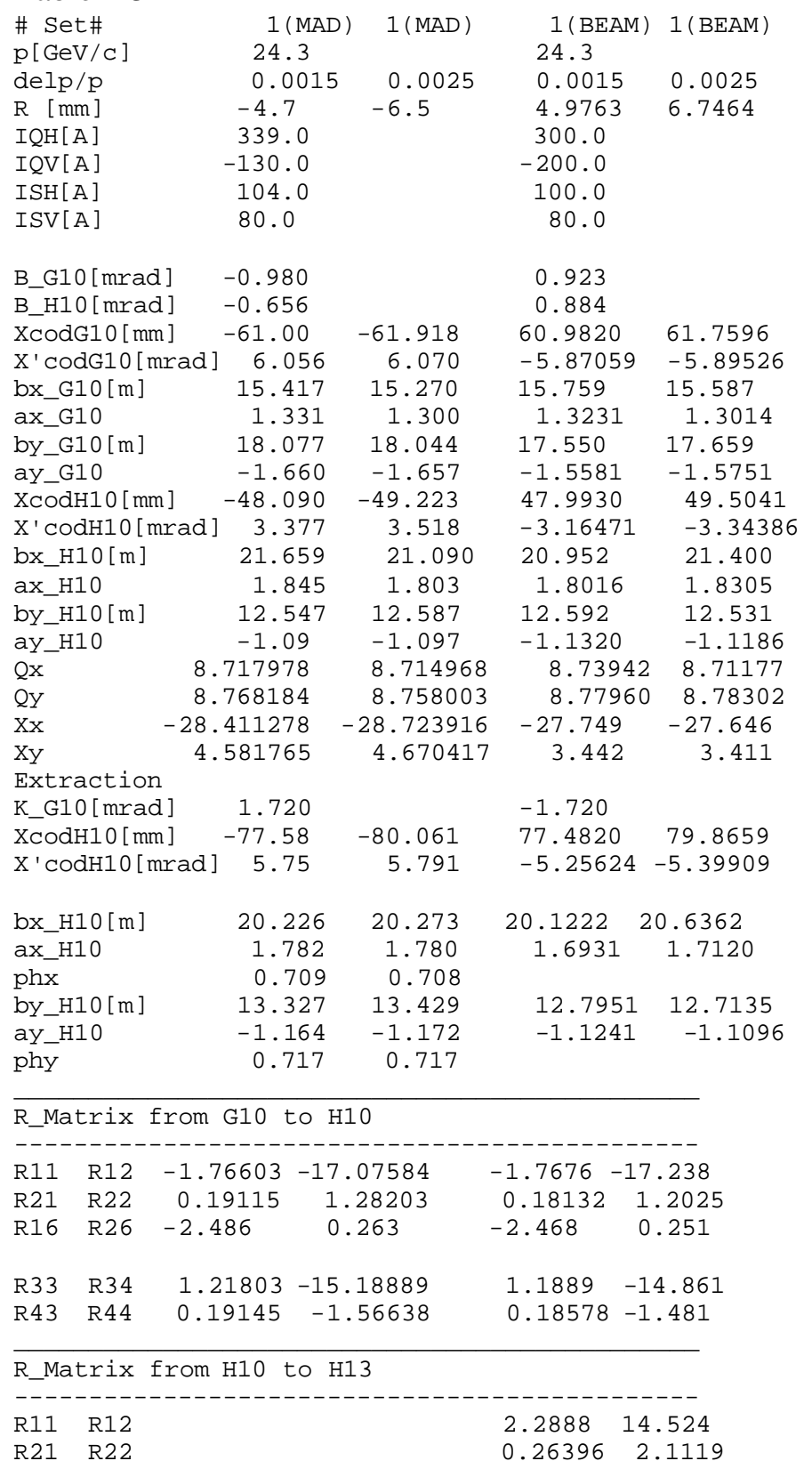




\begin{tabular}{lll} 
R16 R26 & 0.1566 & 0.0156 \\
R33 R34 & 0.050865 & 6.2678 \\
R43 R44 & -0.15793 & 0.19918 \\
\hline Beam Parameters at H13 & \\
----------------------- \\
bx_H13 [m] & 33.6497 \\
ax_H13 & -3.3877 \\
ex[m] & -1.212 \\
e'x & -0.0915 \\
by_H13 [m] & 7.6960 \\
ay_H13 & 0.9899
\end{tabular}

\section{Table X6}

\begin{tabular}{|c|c|c|c|c|}
\hline \# set\# & $1(\mathrm{MAD})$ & 1 (MAD) & 1 (BEAM) & 1 (BEAM) \\
\hline $\mathrm{p}[\mathrm{GeV} / \mathrm{c}]$ & 26.452 & \multicolumn{3}{|c|}{26.452} \\
\hline $\mathrm{delp} / \mathrm{p}$ & 0.0015 & 0.0025 & 0.0015 & 0.0025 \\
\hline $\mathrm{R} \quad[\mathrm{mm}]$ & -4.89 & \multirow[t]{5}{*}{-6.73} & 4.9520 & \multirow{5}{*}{6.74} \\
\hline $\mathrm{IQH}[\mathrm{A}]$ & 330.0 & & 330.0 & \\
\hline$I Q V[A]$ & -210.0 & & -210.0 & \\
\hline $\mathrm{ISH}[\mathrm{A}]$ & 120.0 & & 120.0 & \\
\hline $\operatorname{ISV}[\mathrm{A}]$ & 100.0 & & -100.0 & \\
\hline B_G10 [mrad] & -1.055 & & 0.950 & \\
\hline B_H10 [mrad] & -0.659 & & 0.900 & \\
\hline $\mathrm{XcodG} 10[\mathrm{~mm}]$ & -61.017 & -62.829 & 60.9928 & 61.9493 \\
\hline$X^{\prime}$ codG10 [mrad] & ] 6.041 & 6.147 & -5.84059 & -5.88798 \\
\hline $\mathrm{bx} \_\mathrm{G} 10[\mathrm{~m}]$ & 13.445 & 12.986 & 15.729 & 15.559 \\
\hline ax_G10 & 0.801 & 0.487 & 1.2861 & 1.2542 \\
\hline by_G10 [m] & 17.971 & 17.932 & 18.211 & 18.222 \\
\hline ay_G10 & -1.506 & -1.504 & -1.6390 & -1.6458 \\
\hline $\mathrm{XcodH} 10[\mathrm{~mm}]$ & -47.925 & -49.012 & 47.7788 & 49.4357 \\
\hline $\mathrm{X}^{\prime} \mathrm{codH} 10$ [mrad] & 3.626 & 3.744 & -3.22135 & -3.40253 \\
\hline $\mathrm{bx} \_\mathrm{H} 10[\mathrm{~m}]$ & 28.681 & 38.649 & 22.271 & 22.938 \\
\hline ax_H10 & 2.264 & 3.054 & 1.9121 & 1.9602 \\
\hline by_H10 [m] & 12.004 & 12.029 & 12.246 & 12.272 \\
\hline ay_H10 & -0.976 & -0.980 & -1.1250 & -1.1232 \\
\hline$Q x$ & .583494 & 8.545375 & 8.69804 & 8.66670 \\
\hline Qy & .688998 & 8.696075 & 8.80833 & 8.81539 \\
\hline-36 . & .069879 & -41.783562 & -30.652 & -31.336 \\
\hline Xy & .958385 & 7.047489 & 6.258 & 7.068 \\
\hline \multicolumn{5}{|l|}{ Extraction } \\
\hline K_G10 [mrad] & 1.780 & & -1.720 & \\
\hline $\mathrm{XcodH} 10[\mathrm{~mm}]$ & -77.569 & -80.061 & 77.5316 & 80.1205 \\
\hline $\mathrm{X}^{\prime} \mathrm{codH} 10$ [mrad] & 5.428 & 5.791 & -5.25509 & -5.50674 \\
\hline $\mathrm{bx} \_\mathrm{H} 10[\mathrm{~m}]$ & 27.059 & 20.273 & 21.8586 & 22.8062 \\
\hline ax_H10 & 2.294 & 1.780 & 1.8184 & 1.8489 \\
\hline phx & 0.669 & 0.708 & & \\
\hline by_H10 [m] & 12.433 & 13.429 & 12.5977 & 12.5144 \\
\hline ay_H10 & -1.002 & -1.172 & -1.1141 & -1.0921 \\
\hline phy & 0.712 & 0.717 & & \\
\hline
\end{tabular}

\begin{tabular}{|c|c|c|c|c|c|}
\hline \multicolumn{6}{|c|}{ R_Matrix from G10 to $\mathrm{H} 10$} \\
\hline R11 & $\mathrm{R} 12$ & -1.68356 & -16.65640 & -1.8261 & -17.443 \\
\hline R21 & R22 & 0.16805 & 1.06863 & 0.17912 & 1.1634 \\
\hline $\mathrm{R} 16$ & R2 6 & -2.385 & 0.267 & -2.5889 & 0.2517 \\
\hline R33 & R34 & 1.02039 & -14.523651 & 1.12425 & -15.01 \\
\hline R43 & R44 & 0.17107 & -1.45482 & 0.18951 & -1.48 \\
\hline \multicolumn{6}{|c|}{ R_Matrix from $\mathrm{H} 10$ to $\mathrm{H} 13$} \\
\hline R11 & R12 & & & 2.2886 & 14.523 \\
\hline R21 & R22 & & & 0.26387 & 2.1114 \\
\hline R16 & R26 & & & 0.1566 & 0.0156 \\
\hline
\end{tabular}




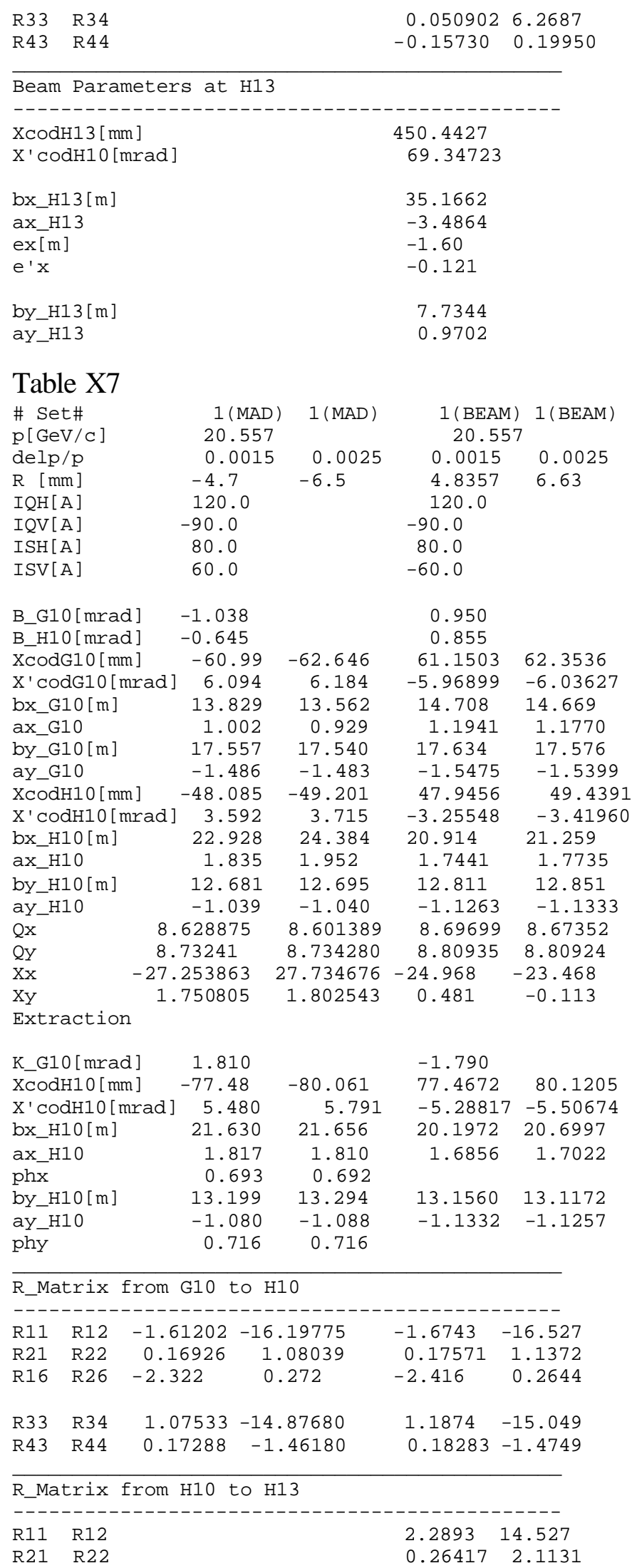




\begin{tabular}{lcc} 
R16 R26 & 0.1568 & 0.0155 \\
R33 R34 & 0.050816 & 6.2658 \\
R43 R44 & -0.15799 & 0.1984 \\
& & \\
\hline Beam Parameters at H13 & & \\
----------- & \\
XcodH13 [mm & 450.1035 \\
X'codH10 [mrad] & 69.30331 \\
bx_H13 [m] & 33.8736 \\
ax_H13 & -3.4299 \\
ex[m] & -1.35 \\
e'x & -0.096 \\
by_H13 [m] & 7.5726 \\
ay_H13 & 1.0001 &
\end{tabular}






